Jonathan S. Lee*, Sarah Lisker, Eric Vittinghoff, Roy Cherian, David B. McCoy, Alex Rybkin, George Su and Urmimala Sarkar

\title{
Follow-up of incidental pulmonary nodules and association with mortality in a safety-net cohort
}

https://doi.org/10.1515/dx-2019-0008

Received February 12, 2019; accepted April 13, 2019; previously published online May 9, 2019

\section{Abstract}

Background: Though incidental pulmonary nodules are common, rates of guideline-recommended surveillance and associations between surveillance and mortality are unclear. In this study, we describe adherence (categorized as complete, partial, late and none) to guideline-recommended surveillance among patients with incidental 5-8 $\mathrm{mm}$ pulmonary nodules and assess associations between adherence and mortality.

Methods: This was a retrospective cohort study of 551 patients ( $\geq 35$ years) with incidental pulmonary nodules conducted from September 1, 2008 to December 31, 2016, in an integrated safety-net health network.

Results: Of the 551 patients, 156 (28\%) had complete, 87 (16\%) had partial, 93 (17\%) had late and 215 (39\%) had no documented surveillance. Patients were followed for a median of 5.2 years [interquartile range (IQR), 3.6-6.7 years] and $82(15 \%)$ died during follow-up. Adjusted all-cause mortality rates ranged from 2.24 [95\% confidence interval

*Corresponding author: Jonathan S. Lee, MD, MAS, Division of General Internal Medicine, University of California, 1545 Divisadero Street, $2^{\text {nd }}$ Floor, San Francisco, CA 94143-0320, USA, Phone: +415-353-7900, Fax: 415-514-8666,

E-mail: jonathan.lee2@ucsf.edu

Sarah Lisker, Roy Cherian and Urmimala Sarkar: Center for Vulnerable Populations, University of California, San Francisco, CA 94143-0320, USA, E-mail: sarah.lisker@ucsf.edu (S. Lisker); roy.cherian@ucsf.edu (R. Cherian); urmimala.sarkar@ucsf.edu (U. Sarkar)

Eric Vittinghoff: Department of Epidemiology and Biostatistics, University of California, San Francisco, CA 94143-0320, USA, E-mail: eric.vittinghoff@ucsf.edu

David B. McCoy and Alex Rybkin: Department of Radiology and Biomedical Imaging, University of California, San Francisco, CA 94143-0320, USA, E-mail: david.mccoy@ucsf.edu (D. B. McCoy); alex.rybkin@ucsf.edu (A. Rybkin)

George Su: Division of Pulmonary, Critical Care, Allergy and Sleep Medicine, University of California, San Francisco, CA 94143-0320, USA, E-mail: george.su@ucsf.edu
(CI), 1.24-3.25] deaths per 100 person-years for complete follow-up to 3.30 (95\% CI, 2.36-4.23) for no follow-up. In multivariable models, there were no statistically significant associations between the levels of surveillance and mortality ( $p>0.16$ for each comparison with complete surveillance). Compared with complete surveillance, adjusted mortality rates were non-significantly increased by 0.45 deaths per 100 person-years ( $95 \%$ CI, -1.10 to 2.01 ) for partial, 0.55 ( $95 \%$ CI, -1.08 to 2.17) for late and 1.05 ( $95 \%$ CI, -0.35 to 2.45 ) for no surveillance.

Conclusions: Although guideline-recommended surveillance of small incidental pulmonary nodules was incomplete or absent in most patients, gaps in surveillance were not associated with statistically significant increases in mortality in a safety-net population.

Keywords: adherence; ambulatory care; care management; diagnosis; guidelines; underserved populations.

\section{Introduction}

Incidental pulmonary nodules are an increasingly common finding in clinical practice with estimates of 1.5 million new cases each year in the United States [1]. The Fleischner Society guidelines recommend longitudinal non-urgent radiographic surveillance for indeterminate pulmonary nodules to document stability, and identify and accurately diagnose nodules which may harbor malignancy $[2,3]$. Surveillance can extend up to 2 years, depending on the nodule size and patient characteristics. While revised guidelines [3] published in 2017 recommend less aggressive surveillance of pulmonary nodules, prior and more aggressive Fleischner guidelines [2] were the standard for clinical practice from 2005 through 2017. Though most incidental nodules are benign, failure to provide appropriate and timely longitudinal follow-up can lead to delayed cancer diagnoses, poor patient outcomes and costly malpractice claims $[4,5]$.

Incidental pulmonary nodules present unique barriers to longitudinal evaluation and care coordination. By definition, these nodules are detected on radiographic imaging ordered for unrelated reasons such as evaluation 
of trauma or pulmonary embolism [6-8]. It follows that incidental nodules are often discovered in acute care hospital settings where the physician ordering the study is unlikely to be the physician following up the incidental findings once the patient leaves the hospital. Responsibility for longitudinal surveillance generally falls on the primary care physician, and prior studies demonstrate that communication of test results is often inadequate during the transition from acute to outpatient settings [9-11].

The few studies [6, 12] that have assessed the adequacy of guideline-adherent nodule surveillance in usual care settings demonstrate highly variable results, and little is known about the levels of adherence to Fleischner guidelines or associations between adherence and patient outcomes such as mortality, where earlier detection and treatment of lung cancer could potentially have an impact. In addition, rates of adherence in safetynet health systems, where patients may be at higher risk for lung cancer compared with the general population due to higher smoking rates, are unclear. Patient characteristics such as limited English proficiency and health literacy, which are more prevalent among patients cared for in safety-net settings, and limited institutional resources may further complicate longitudinal care. Thus, we aimed to evaluate the levels and rates of adherence to guidelinerecommended surveillance and associations between guideline adherence and mortality in a safety-net cohort of patients with incidental pulmonary nodules.

\section{Subjects and methods}

\section{Study design}

We performed a retrospective cohort study of patients with indeterminate incidental pulmonary nodules. We queried our health system's radiology database to identify eligible patients and reviewed medical records to collect patient and clinical data. The University of California, San Francisco Institutional Review Board reviewed and approved this study (15-18436).

\section{Setting}

The study took place within a publicly funded, urban, integrated health network which includes the largest safety-net hospital in San Francisco, serving approximately 106,000 patients annually, with nearly 600,000 outpatient visits. The patient population is predominately publicly insured or uninsured and racially and ethnically diverse with the majority of patients being Hispanic, Asian and nonHispanic African-American. Patients cared for in the San Francisco Health Network (SFHN) experience a higher prevalence of homelessness, psychiatric comorbidity and smoking compared to the general state population [13].

\section{Participants}

We included adults aged 35 years and older with 5-8 mm pulmonary nodules discovered incidentally between September 1, 2008 and December 31, 2014 for whom guidelines recommend longitudinal computed tomography (CT) surveillance. We chose 2008 as the initial time point because the hospital had recently installed new CT scanners and it provides a 3-year buffer period for the dissemination of recommendations following the publication of Fleischner guidelines for nodule surveillance in 2005. We assessed adherence to the 2005 guidelines [2] as opposed to more recent guidelines [3] because the 2005 guidelines were applicable during the study period. The 2005 Fleischner guidelines recommend follow-up CT scans within at least 1 year for all patients with nodules of this size. We chose the age cutoff to match the guideline criteria and the end time point to allow for at least 2 years of follow-up. We excluded patients with smaller nodules ( $4 \mathrm{~mm}$ or less) that would not necessarily require followup and larger nodules (greater than $8 \mathrm{~mm}$ ) that would likely trigger immediate referral for advanced studies such as positron-emission tomography (PET) scans and biopsies based on guideline recommendations. We excluded patients with a history of active or previous cancer for whom guidelines would not apply, patients who died within 3 months of the index CT scan and patients without any primary care, specialty care or non-trauma emergency department visits with the health system within 24 months after the index scan. We aimed to evaluate surveillance for patients engaged with the health system who would represent true missed opportunities for appropriate follow-up. To our knowledge, no systematic interventions to track, communicate or improve pulmonary nodule surveillance were implemented or in effect during the study period, and no standard institutional guidelines for radiologists' reporting of recommended surveillance existed.

To identify eligible patients, we searched all CT radiology reports within the Radiology Information System database for the keyword "nodule" using the Microsoft SQL Server Reporting Services software (Redmond, WA, USA). We developed a natural language processing (NLP) tool in Python (Wilmington, DE, USA) as an initial screen to identify the nodule size and to exclude non-pulmonary nodules and CT scans performed for malignancy staging. We performed a medical chart review of the remaining patients to confirm eligibility.

\section{Data collection}

We queried the electronic health record to obtain structured patient data including sex, race/ethnicity and primary language. We developed an abstraction protocol and standardized the data collection form using REDCap (version 8) and performed a chart review to capture patient (history of smoking, homelessness and psychiatric comorbidity) and nodule (size, upper lobe location, consistency and reason for exam) characteristics, and nodule evaluation including CT scans, PET scans and biopsies. We abstracted radiology recommendations for surveillance and reviewed primary care notes following the index scan, when available, abstracting medical decision making to not pursue the recommended follow-up. We abstracted nodule evaluation until reaching one of the following endpoints: (1) nodule stability as defined by Fleischner guidelines or radiology recommendation that no further follow-up was needed; (2) diagnosis of lung or other cancer; (3) patient death or 
lost to follow-up from the health system or (4) documentation of a decision to not pursue follow-up. Five trained researchers performed medical record abstraction. Two researchers abstracted each record until they reached an inter-rater agreement of 0.88 , measured by Cohen's Kappa coefficient. Discrepancies in coding were resolved by consensus among senior study investigators. We excluded patients who were found to meet previously defined exclusion criteria during medical record review.

\section{Categorization of nodule surveillance}

We categorized patients as high risk if they were current or former smokers. We treated ground glass nodules as solid nodules because the 2005 Fleischner guidelines do not give a specific recommendation for the duration of follow-up for sub-solid nodules. We defined evaluation as "complete" if all recommended CT/PET scans or biopsies were completed and timely, and a nodule stability or cancer diagnosis endpoint was reached. We defined scans as timely if they occurred within 3 months of recommended follow-up at 6-12 months and within 2 months for recommended follow-up at 3-6 months to allow for scheduling and other delays encountered in usual care, similar to definitions used by previous studies $[6,14]$. We defined evaluation as "partial" if the first follow-up scan was completed and timely but any subsequent recommended evaluation was not completed or not timely. We defined evaluation as "late" if the first follow-up scan was completed but was not timely regardless of subsequent evaluation. We categorized patients who had no surveillance evaluation as "none."

\section{Patient outcomes}

We abstracted cancer diagnoses and death from the medical record. To account for patients lost to follow-up or no longer cared for in the SFHN, we supplemented our analysis using mortality and cause-ofdeath data through 2016 from the National Center for Health Statistics' National Death Index.

\section{Statistical analysis}

We used multiple imputation by iterative chained equations to impute missing data [15]. Using the resulting 20 completed datasets, we used logistic and multinomial models to compare baseline patient characteristics across follow-up categories, combining results according to Rubin's rules [16]. In addition, for each category, we calculated unadjusted mortality rates per 100 person-years and then used a Poisson model [17], with the log time from the index scan until death or the end of 2016 as an offset, to estimate the adjusted between-category mortality rate ratios, controlling for patient and nodule characteristics. Regression standardization was then used to obtain marginal adjusted rates and rate differences, based on the Poisson model. In two sensitivity analyses, we first repeated the assessment of between-category differences using Cox models, to check sensitivity to the Poisson assumption of a constant baseline mortality rate, and then repeated the Poisson analysis using observed complete-case data. For both sensitivity analyses, results were similar to the primary multiple imputation analysis and are not reported. We performed all statistical analyses using Stata 15.1 (StataCorp LLC, College Station, TX, USA). Two-sided p-values $<0.05$ were considered statistically significant.

\section{Results}

\section{Study population and nodule surveillance}

Our NLP algorithm identified 1365 patients with possible pulmonary nodules detected on CT scans during the study period. We excluded 666 patients after initial review, primarily related to nodule size and patients who were not engaged in care within 24 months of the index scan (Figure 1). We conducted a complete medical record review of the remaining 699 patients and excluded 148 for a final cohort of 551 patients with 5-8 mm pulmonary nodules requiring follow-up. We categorized 156 (28\%) patients as complete, $87(16 \%)$ as partial, $93(17 \%)$ as late and $215(39 \%)$ as no surveillance.

Overall, the mean age was 59 years [standard deviation (SD) 11], 58\% of patients were male and over half (58\%) were non-Hispanic African-American, Hispanic or Asian (Table 1). Most patients $(66 \%)$ were current or former smokers. Over one-quarter (27\%) of patients had a history of homelessness, $40 \%$ had a history of substance abuse and 34\% had a history of psychiatric illness. Nearly three-quarters of nodules (72\%) were 5-6 $\mathrm{mm}$ in size and $39 \%$ were located in the upper lobes of the lung. Nearly two-thirds of patients (61\%) had multiple nodules on index CT scans.

The average number of surveillance CT scans was 2.2 for complete, 3.1 for partial and 1.8 for late follow-up groups. For patients with complete surveillance, two (1\%) were diagnosed with lung cancer during follow-up. Among those with less-than-complete surveillance, seven (2\%) were diagnosed with lung cancer during follow-up. For patients with partial surveillance, the vast majority $(85,98 \%)$ completed two or more surveillance scans while only two (2\%) patients had no further surveillance after the first follow-up scan. For patients with late surveillance, 51\% with 5-6 mm nodules had a delay of at least 24 months (vs. guideline-recommended 6-12 months for high or low risk), $67 \%$ of low-risk patients with $7-8 \mathrm{~mm}$ nodules had a delay of at least 24 months (guideline-recommended 6-12 months) and $82 \%$ of high-risk patients with 7-8 mm nodules had a delay of at least 12 months (guideline-recommended 3-6 months) between the index and first surveillance scan. 


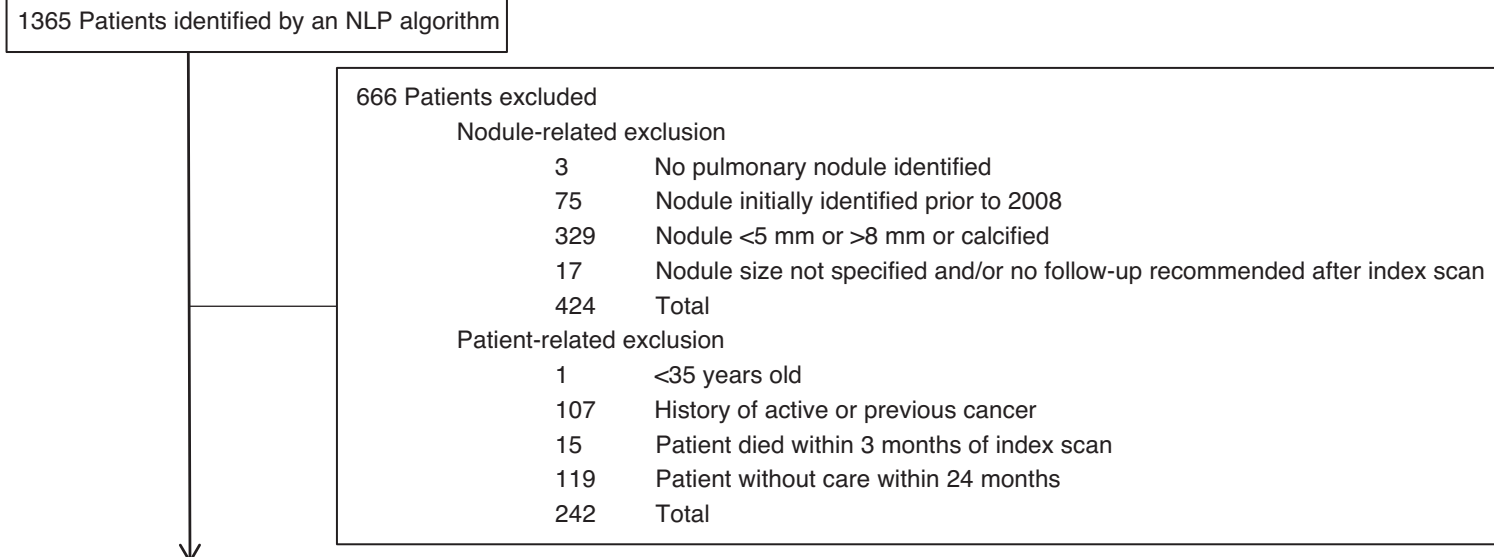

699 Patient records abstracted

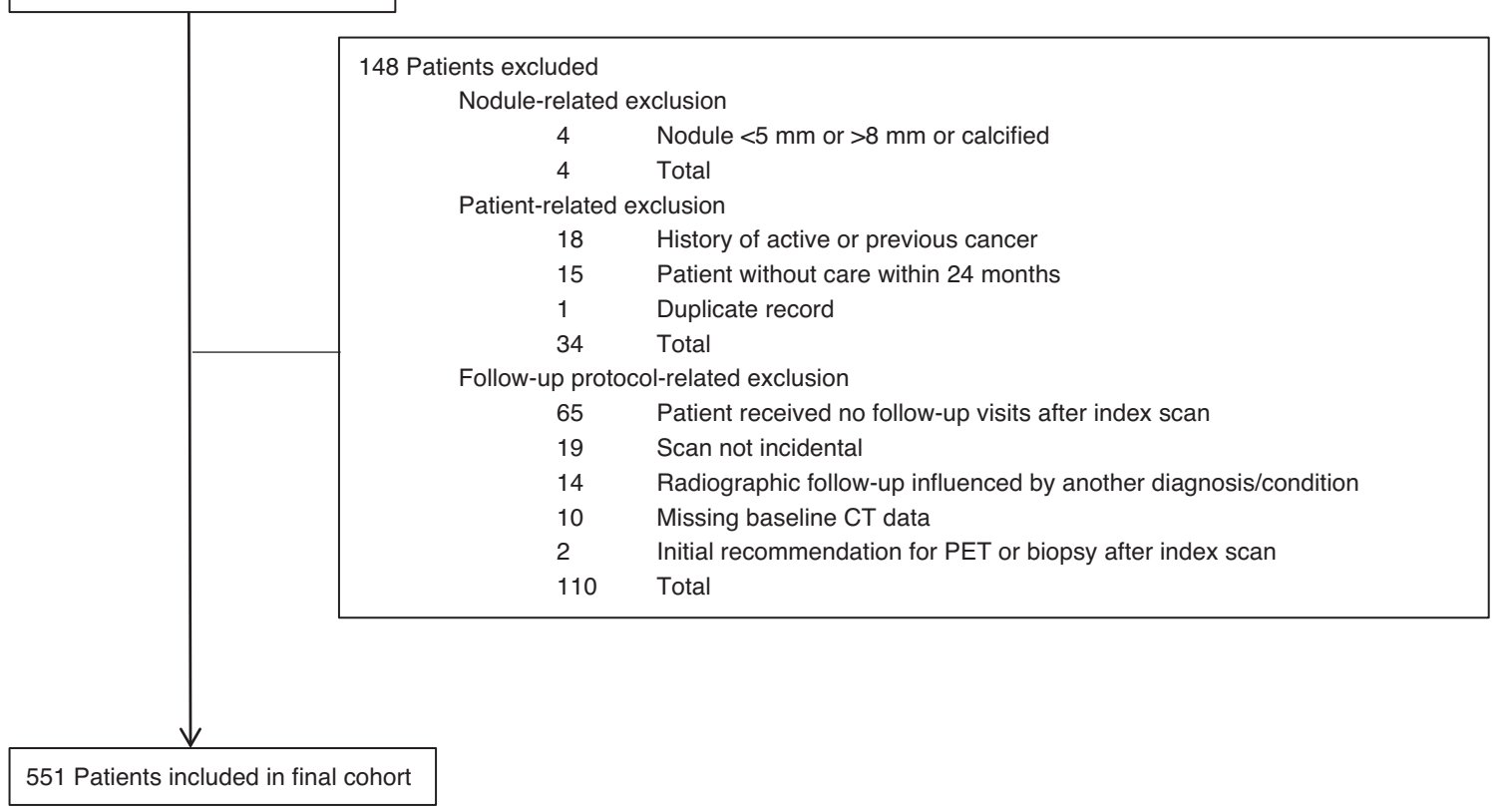

Figure 1: Patient identification and selection.

NLP, natural language processing.

\section{Association between nodule follow-up and mortality}

In multivariable regression models, there were no statistically significant associations between the levels of surveillance and mortality ( $p>0.16$ for each comparison with complete surveillance). The adjusted mortality rate ratios were non-significantly elevated for partial [adjusted rate ratio (ARR) 1.20; 95\% confidence interval (CI), 0.64-2.25], late (ARR 1.24; 95\% CI, 0.65-2.36) and no (ARR 1.47; 95\% CI, 0.86-2.52) surveillance when compared with complete surveillance (Table 2). A nodule size of 7-8 mm (vs. 5-6 mm) was associated with greater mortality (ARR 2.19; 95\% CI, 1.51-3.20).

\section{Mortality rates and differences by nodule follow-up}

Patients were followed for a median of 5.2 years [interquartile range (IQR), 3.6-6.7 years], and 82 (15\%) died during follow-up. There were few deaths attributed specifically to lung cancer (one in each surveillance group) based on the chart review and the National Death Index cause of death data. Adjusted all-cause mortality rates were similar to unadjusted rates and ranged from 2.24 (95\% CI, 1.24-3.25) deaths per 100 person-years for complete follow-up to 3.30 ( $95 \%$ CI, 2.36-4.23) deaths per 100 person-years for no follow-up (Table 3). Compared with complete surveillance, adjusted mortality rates were 
Table 1: Characteristics of patients and baseline incidental pulmonary nodules stratified by the level of surveillance. ${ }^{a}$

\begin{tabular}{|c|c|c|c|c|c|}
\hline & \multicolumn{5}{|c|}{ Level of surveillance $^{b}$} \\
\hline & $\begin{array}{r}\text { Complete } \\
n=156\end{array}$ & $\begin{array}{r}\text { Partial } \\
n=87\end{array}$ & $\begin{array}{r}\text { Late } \\
n=93\end{array}$ & $\begin{array}{r}\text { None } \\
n=215\end{array}$ & p-Value \\
\hline \multicolumn{6}{|l|}{ Patient characteristics } \\
\hline \multicolumn{6}{|l|}{ Demographics } \\
\hline Age, years, mean (SD) & $60(9)$ & $56(11)$ & $57(9)$ & $59(13)$ & 0.02 \\
\hline Male, $\%$ & 51 & 60 & 62 & 61 & 0.17 \\
\hline Race and ethnicity, \% & & & & & 0.63 \\
\hline White, non-Hispanic & 27 & 30 & 25 & 27 & \\
\hline Black, non-Hispanic & 20 & 25 & 29 & 24 & \\
\hline Hispanic & 6 & 1 & 2 & 5 & \\
\hline Asian & 33 & 30 & 24 & 31 & \\
\hline Other ${ }^{c}$ & 14 & 14 & 20 & 14 & \\
\hline History of homelessness, \% & 21 & 22 & 34 & 30 & 0.13 \\
\hline Smoking history, \% & & & & & 0.16 \\
\hline Never smoker & 38 & 31 & 25 & 36 & \\
\hline Former smoker & 26 & 29 & 20 & 25 & \\
\hline Current smoker & 36 & 40 & 55 & 39 & \\
\hline History of substance abuse, \% & 33 & 39 & 50 & 42 & 0.09 \\
\hline History of psychiatric illness, \% & & & & & 0.22 \\
\hline None & 69 & 58 & 62 & 70 & \\
\hline Anxiety or depression & 24 & 35 & 28 & 20 & \\
\hline Schizophrenia & 7 & 8 & 10 & 10 & \\
\hline \multicolumn{6}{|l|}{ Nodule characteristic } \\
\hline Nodule size, mm, \% & & & & & 0.16 \\
\hline $5-6$ & 72 & 63 & 79 & 72 & \\
\hline $7-8$ & 28 & 37 & 22 & 28 & \\
\hline Upper lobe location, \% & 39 & 39 & 37 & 41 & 0.93 \\
\hline Ground glass, \% & 5 & 5 & 2 & 2 & 0.56 \\
\hline Multiple nodules, \% & 69 & 66 & 63 & 52 & 0.005 \\
\hline Reason for initial chest CT, \% & & & & & $<0.001$ \\
\hline Pulmonary embolism evaluation & 7 & 15 & 14 & 10 & \\
\hline Trauma & 1 & 2 & 7 & 15 & \\
\hline Concern for infection & 7 & 14 & 15 & 12 & \\
\hline Weight loss & 6 & 1 & 1 & 1 & \\
\hline Chest pain & 5 & 6 & 8 & 9 & \\
\hline General shortness of breath & 6 & 8 & 9 & 10 & \\
\hline Nodule seen on other imaging & 47 & 33 & 20 & 23 & \\
\hline Chronic obstructive pulmonary disease & 5 & 6 & 3 & 2 & \\
\hline Concern for aortic aneurysm or dissection & 3 & 4 & 9 & 5 & \\
\hline Other & 13 & 12 & 15 & 12 & \\
\hline
\end{tabular}

CT, computed tomography; SD, standard deviation. a Group totals may not sum to $100 \%$ due to imputation and rounding. Data were missing from the complete 551-patient cohort and imputed for the following variables, reported as missing $n$ (\%): male 7 (1), race/ethnicity 13 (2), smoking history 113 (21), homelessness history 119 (22), substance abuse history 104 (19) and ground glass nodule 7 (1). b' defined surveillance as "complete" if all recommended testing was completed and timely, "partial" if the first follow-up CT scan was completed and timely but any subsequent recommended evaluation was not completed or not timely, "late" if the first follow-up scan was completed but was not timely regardless of subsequent evaluation, and "none" if patients had no follow-up evaluation. We defined scans as timely if they occurred within 2 or 3 months of the recommended follow-up time period depending on the specific time period. 'Other race and ethnicity consisted of patients whose race was reported as Native American or Alaskan Native.

non-significantly increased by 0.45 deaths per 100 person-years (95\% CI, -1.10 to 2.01) for partial, 0.55 (95\% CI, -1.08 to 2.17 ) for late and 1.05 ( $95 \% \mathrm{CI},-0.35$ to 2.45 ) for no surveillance.

\section{Discussion}

In this study of 551 patients with incidental 5-8 mm pulmonary nodules, we found significant gaps in 
Table 2: Adjusted all-cause mortality rate ratios by the level of adherence and patient and nodule characteristics. ${ }^{a}$

\begin{tabular}{|c|c|}
\hline Level of surveillance $^{b}$ & $\begin{array}{l}\text { Adjusted mortality } \\
\text { rate ratio }(95 \% \mathrm{Cl})\end{array}$ \\
\hline Complete & Reference \\
\hline Partial & $1.20(0.64-2.25)$ \\
\hline Late & $1.24(0.65-2.36)$ \\
\hline None & $1.47(0.86-2.52)$ \\
\hline \multicolumn{2}{|l|}{ Patient characteristics } \\
\hline Age, per 10 years & $1.37(1.16-1.62)$ \\
\hline Male & $1.13(0.71-1.81)$ \\
\hline \multicolumn{2}{|l|}{ Race and ethnicity } \\
\hline White, non-Hispanic & Reference \\
\hline Black, non-Hispanic & $1.23(0.79-1.92)$ \\
\hline Hispanic & $1.71(0.89-3.28)$ \\
\hline Asian & $0.48(0.23-1.02)$ \\
\hline Other & $0.54(0.23-1.24)$ \\
\hline History of homelessness & $0.80(0.48-1.34)$ \\
\hline \multicolumn{2}{|l|}{ Smoking history } \\
\hline Never smoker & Reference \\
\hline Former smoker & $1.57(0.69-3.56)$ \\
\hline Current smoker & $1.58(0.74-3.33)$ \\
\hline History of substance abuse & $1.33(0.77-2.30)$ \\
\hline \multicolumn{2}{|l|}{ History of psychiatric illness } \\
\hline None & Reference \\
\hline Anxiety or depression & $1.38(0.86-2.21)$ \\
\hline Schizophrenia & $1.50(0.74-3.02)$ \\
\hline \multicolumn{2}{|l|}{ Nodule characteristic } \\
\hline \multicolumn{2}{|l|}{ Nodule size, mm } \\
\hline $5-6$ & Reference \\
\hline $7-8$ & $2.19(1.51-3.20)$ \\
\hline Upper lobe location & $1.21(0.82-1.80)$ \\
\hline Ground glass & $1.64(0.65-4.16)$ \\
\hline Multiple nodules & $0.85(0.57-1.28)$ \\
\hline
\end{tabular}

$\mathrm{Cl}$, confidence interval. ${ }^{\text {TThe }}$ adjusted rate ratios were calculated using Poisson models adjusted for patient (age, sex, and race/ ethnicity, and history of smoking, substance abuse and psychiatric illness) and nodule (size, upper lobe location, ground glass and multiple) characteristics. "We defined surveillance as "complete" if all recommended testing was completed and timely, "partial" if the first follow-up CT scan was completed and timely but any subsequent recommended evaluation was not completed or not timely, "late" if the first follow-up scan was completed but was not timely regardless of subsequent evaluation, and "none" if patients had no follow-up evaluation. We defined scans as timely if they occurred within 2 or 3 months of the recommended follow-up time period depending on the specific time period. 'Other race and ethnicity consisted of patients whose race was reported as Native American or Alaskan Native.

recommended surveillance with only $28 \%$ of patients completing all follow-up and 39\% with no documented follow-up. However, gaps in surveillance were not associated with statistically significant differences in mortality in this safety-net population. Stated another way, complete adherence to guidelines was not associated with reduced mortality. To our knowledge, this is one of few studies of usual care settings to assess the completeness of adherence to pulmonary nodule guidelines and the only study to assess associations between adherence and mortality.

Our finding of large gaps in pulmonary nodule surveillance is consistent with existing studies evaluating follow-up for patients in usual care settings. In two single-center studies, only $29 \%$ and $48 \%$ of patients completed at least one recommended surveillance scan $[6,12]$. However, neither of these studies assessed surveillance up to nodule stability or diagnosis of lung cancer. A study conducted within the Veterans' Administration health system found that $92 \%$ of patients underwent some degree of nodule surveillance with $11 \%$ of patients undergoing surveillance across multiple facilities [18]. These impressive follow-up rates may not be generalizable to other usual care settings, such as in this study, that lack a standard electronic health record accessible by any facility within the integrated health system.

Despite a high proportion of patients with incomplete or no surveillance, we found no associations between the completeness of surveillance and mortality. One likely explanation for this discrepancy is that deaths from lung cancer were outweighed by deaths from other causes. Amongst patients in our study with complete follow-up, only $1 \%$ were diagnosed with lung cancer, a lower proportion than other studies evaluating nodules 5-8 mm in size $[5,18,19]$. Larger nodule size, which is associated with increased lung cancer risk, was also associated with increased mortality in our study. However, few deaths in any group were attributed to lung cancer. It is possible that the level of surveillance may be associated with reduced mortality amongst patients diagnosed with lung cancer due to earlier detection but we were not able to assess this. A longer duration of follow-up may also be required to detect associations between nodule surveillance and mortality. The National Lung Screening Trial [20] followed high-risk patients for a median of 6.5 years to demonstrate reduced lung cancer and all-cause mortality compared with a median follow-up of 5.2 years in our study.

Our study has important implications for patients, clinicians and health systems. First, though validation of the 2005 Fleischner guidelines was not our primary objective, our findings support a less aggressive approach to surveillance of pulmonary nodules $5-8 \mathrm{~mm}$ in size than recommended by these guidelines given the demonstrated lack of association between follow-up and mortality. Accordingly, the 2017 Fleischner guidelines recommend no routine surveillance for nodules less than $6 \mathrm{~mm}$ in size and a less aggressive surveillance of high-risk patients 
Table 3: Unadjusted and adjusted all-cause mortality rates by the level of surveillance.

Mortality rate per 100 person-years $(95 \% \mathrm{Cl})$

\begin{tabular}{lrrr}
\hline Level of surveillance $^{\mathrm{a}}$ & Unadjusted & Adjusted $^{\mathrm{b}}$ & Adjusted difference $^{\mathrm{b}}$ \\
\hline Complete & $2.22(1.23-3.21)$ & $2.24(1.24-3.25)$ & Reference \\
Partial & $2.84(1.56-4.11)$ & $2.70(1.50-3.89)$ & $0.45(-1.10$ to 2.01$)$ \\
Late & $2.64(1.39-3.90)$ & $2.79(1.52-4.06)$ & $0.55(-1.08$ to 2.18$)$ \\
None & $3.50(2.48-4.51)$ & $3.30(2.36-4.23)$ & $1.05(-0.35$ to 2.45$)$ \\
\hline
\end{tabular}

$\mathrm{Cl}$, confidence interval. "We defined surveillance as "complete" if all recommended testing was completed and timely, "partial" if the first follow-up CT scan was completed and timely but any subsequent recommended evaluation was not completed or not timely, "late" if the first follow-up scan was completed but was not timely regardless of subsequent evaluation, and "none" if patients had no follow-up evaluation. We defined scans as timely if they occurred within 2 or 3 months of the recommended follow-up time period depending on the specific time period. ${ }^{b}$ Mortality rates were adjusted for patient (age, sex and race/ethnicity, and history of smoking, substance abuse and psychiatric illness) and nodule (size, upper lobe location, ground glass and multiple) characteristics. Adjusted difference is the difference between mortality rates in comparison to the complete surveillance group.

with 7-8 mm pulmonary nodules. Prospective studies are needed to validate the impact of these updated guidelines on patient outcomes. Second, while the 2017 Fleischner guidelines are likely to significantly reduce the burden of recommended surveillance CT scans for patients and clinicians, adherence to new guidelines is still likely to be poor in usual care settings unless effective tracking and notification systems are established [21, 22]. These systems will be increasingly important to monitor guideline concordance as the United States Preventive Services Task Force recommendation for lung cancer screening [23] and the newer Fleischner guidelines, which allow clinicians greater freedom and discretion in determining the need for surveillance, are integrated into clinical practice. Achieving high levels of adherence will also require understanding challenges that clinicians face when managing abnormal results [24-26] and improving communication of subcritical results requiring follow-up during transitions of care [10, 27]. Third, the uncertainties regarding the impact of adherence to Fleischner guidelines on significant patient outcomes emphasize the importance of shared decision-making between patient and clinician regarding the risks and benefits of nodule evaluation [28, 29]. Educational strategies to increase clinician knowledge and improve communication skills may support shared decision-making conversations and improve adherence [30-32]. Finally, our study demonstrates the importance of measuring guideline adherence and its impact on patient outcomes in real-world settings to validate practice guidelines, consistent with research mandates for pulmonary nodules from the American Thoracic Society [33]. This type of observational research is relevant across a spectrum of common findings with guideline-recommended surveillance and variable clinical significance such as adrenal incidentalomas and abnormal cervical cancer screening results and has the potential to change the intensity of guideline recommendations for surveillance [34, 35].

Our study has limitations. First, the low proportion of incident lung cancer diagnoses limits our ability to detect differences in all-cause and lung cancer-related mortality between follow-up groups. Second, findings in a safety-net population may not be generalizable to other populations given the patient diversity and high prevalence of comorbid homelessness, substance abuse and psychiatric illness. Third, we cannot account for any follow-up testing that was done at outside facilities, which could alter our estimates of follow-up completeness. Fourth, we could not fully ascertain diagnoses of lung cancer in patients with incomplete surveillance. Finally, inadequate documentation of clinical reasons for not pursuing follow-up, such as multiple comorbidities, may have led to residual confounding in our observational study.

\section{Conclusions}

In conclusion, adherence to guideline-recommended surveillance of incidental pulmonary nodules was incomplete or completely lacking in most patients but was not associated with mortality in a safety-net population. Further study is needed to identify strategies to improve adherence to and to demonstrate empirical evidence for the effectiveness of pulmonary nodule guidelines.

Acknowledgments: We would like to thank Gato Gourley, Kate Radcliffe, Mekhala Hoskote, Michael Bentz and the UCSF Academic Research Systems. 
Author contributions: All the authors have accepted responsibility for the entire content of this submitted manuscript and approved submission.

Research funding: Dr. Lee was supported by an HRSA grant T32HP19025, Funder Id: http://dx.doi. org/10.13039/100000102. Dr. Sarkar was supported through a grant from the Agency for Healthcare Research and Quality (P3OHS023558), Funder Id: http://dx.doi. org/10.13039/100000133 and the National Cancer Institute (K24CA212294), Funder Id: http://dx.doi. org/10.13039/100000054.

Employment or leadership: None declared. Honorarium: None declared.

Competing interests: The funding organization(s) played no role in the study design; in the collection, analysis, and interpretation of data; in the writing of the report; or in the decision to submit the report for publication.

\section{References}

1. Gould MK, Tang T, Liu IA, Lee J, Zheng C, Danforth KN, et al. Recent trends in the identification of incidental pulmonary nodules. Am J Respir Crit Care Med 2015;192:1208-14.

2. MacMahon H, Austin JH, Gamsu G, Herold CJ, Jett JR, Naidich DP, et al. Guidelines for management of small pulmonary nodules detected on CT scans: a statement from the Fleischner Society. Radiology 2005;237:395-400.

3. MacMahon H, Naidich DP, Goo JM, Lee KS, Leung AN, Mayo $J R$, et al. Guidelines for management of incidental pulmonary nodules detected on CT images: from the Fleischner Society 2017. Radiology 2017;284:228-43.

4. Berlin L. Failure to diagnose lung cancer: anatomy of a malpractice trial. AJR Am J Roentgenol 2003;180:37-45.

5. McWilliams A, Tammemagi MC, Mayo JR, Roberts H, Liu G, Soghrati K, et al. Probability of cancer in pulmonary nodules detected on first screening CT. N Engl J Med 2013;369:910-9.

6. Blagev DP, Lloyd JF, Conner K, Dickerson J, Adams D, Stevens SM, et al. Follow-up of incidental pulmonary nodules and the radiology report. J Am Coll Radiol 2014;11:378-83.

7. Hall WB, Truitt SG, Scheunemann LP, Shah SA, Rivera MP, Parker LA, et al. The prevalence of clinically relevant incidental findings on chest computed tomographic angiograms ordered to diagnose pulmonary embolism. Arch Intern Med 2009;169:1961-5.

8. Hammerschlag G, Cao J, Gumm K, Irving L, Steinfort D. Prevalence of incidental pulmonary nodules on computed tomography of the thorax in trauma patients. Intern Med J 2015;45:630-3.

9. Kripalani S, LeFevre F, Phillips CO, Williams MV, Basaviah P, Baker DW. Deficits in communication and information transfer between hospital-based and primary care physicians: implications for patient safety and continuity of care. J Am Med Assoc 2007;297:831-41.
10. Were MC, Li X, Kesterson J, Cadwallader J, Asirwa C, Khan B, et al. Adequacy of hospital discharge summaries in documenting tests with pending results and outpatient follow-up providers. J Gen Intern Med 2009;24:1002-6.

11. Callen JL, Westbrook JI, Georgiou A, Li J. Failure to follow-up test results for ambulatory patients: a systematic review. J Gen Intern Med 2012;27:1334-48.

12. Ridge CA, Hobbs BD, Bukoye BA, Aronson MD, Boiselle PM, Leffler DA, et al. Incidentally detected lung nodules: clinical predictors of adherence to Fleischner Society surveillance guidelines. J Comput Assist Tomogr 2014;38:89-95.

13. Harder + Company Community Research. Community Health Status Assessment: City and County of San Francisco 2012. Available from: https://www.sfdph.org/dph/files/chip/CommunityHealthStatusAssessment.pdf.

14. McDonald JS, Koo CW, White D, Hartman TE, Bender CE, Sykes AG. Addition of the Fleischner Society guidelines to chest CT examination interpretive reports improves adherence to recommended follow-up care for incidental pulmonary nodules. Acad Radiol 2017;24:337-44.

15. White IR, Royston P, Wood AM. Multiple imputation using chained equations: issues and guidance for practice. Stat Med 2011;30:377-99.

16. Rubin DB. Multiple imputation for nonresponse in surveys. New York: Wiley; 1987.

17. McCullagh P, Nelder JA. Generalized linear models, second ed. Cambridge: Chapman \& Hall; 1989.

18. Wiener RS, Gould MK, Slatore CG, Fincke BG, Schwartz LM, Woloshin S. Resource use and guideline concordance in evaluation of pulmonary nodules for cancer too much and too little care. J Am Med Assoc Intern Med 2014;174:871-80.

19. Henschke Cl, Yankelevitz DF, Naidich DP, McCauley DI, McGuinness G, Libby DM, et al. CT screening for lung cancer: suspiciousness of nodules according to size on baseline scans. Radiology 2004;231:164-8.

20. National Lung Screening Trial Research T, Aberle DR, Adams AM, Berg CD, Black WC, Clapp JD, et al. Reduced lung-cancer mortality with low-dose computed tomographic screening. N Engl J Med 2011;365:395-409.

21. Shelver J, Wendt CH, McClure M, Bell B, Fabbrini AE, Rector $T$, et al. Effect of an automated tracking registry on the rate of tracking failure in incidental pulmonary nodules. J Am Coll Radiol 2017;14:773-7.

22. Lacson R, O'Connor SD, Andriole KP, Prevedello LM, Khorasani R. Automated critical test result notification system: architecture, design, and assessment of provider satisfaction. AJR Am J Roentgenol 2014;203:W491-6.

23. Moyer VA, Force USPST. Screening for lung cancer: U.S. Preventive Services Task Force recommendation statement. Ann Intern Med 2014;160:330-8.

24. Hysong SJ, Sawhney MK, Wilson L, Sittig DF, Espadas D, Davis T, et al. Provider management strategies of abnormal test result alerts: a cognitive task analysis. J Am Med Inform Assoc 2010;17:71-7.

25. Hysong SJ, Sawhney MK, Wilson L, Sittig DF, Esquivel A, Singh S, et al. Understanding the management of electronic test result notifications in the outpatient setting. BMC Med Inform Decis Mak 2011;11:22. 
26. Murphy DR, Reis B, Sittig DF, Singh H. Notifications received by primary care practitioners in electronic health records: a taxonomy and time analysis. Am J Med 2012;125:209 e1-7.

27. Moore C, Wisnivesky J, Williams S, McGinn T. Medical errors related to discontinuity of care from an inpatient to an outpatient setting. J Gen Intern Med 2003;18:646-51.

28. Gould MK, Donington J, Lynch WR, Mazzone PJ, Midthun DE, Naidich DP, et al. Evaluation of individuals with pulmonary nodules: when is it lung cancer? Diagnosis and management of lung cancer, 3rd ed: American College of Chest Physicians Evidence-Based Clinical Practice Guidelines. Chest 2013;143:E93-120.

29. Wiener RS, Slatore CG, Gillespie C, Clark JA. Pulmonologists' reported use of guidelines and shared decision-making in evaluation of pulmonary nodules a qualitative study. Chest 2015;148:1415-21.

30. Golden SE, Wiener RS, Sullivan D, Ganzini L, Slatore CG. Primary care providers and a system problem: a qualitative study of clinicians caring for patients with incidental pulmonary nodules. Chest 2015;148:1422-9.

31. Freiman MR, Clark JA, Slatore CG, Gould MK, Woloshin S, Schwartz LM, et al. Patients' knowledge, beliefs, and distress associated with detection and evaluation of incidental pulmonary nodules for cancer: results from a multicenter survey. J Thorac Oncol 2016;11:700-8.

32. Slatore CG, Wiener RS. Pulmonary nodules: a small problem for many, severe distress for some, and how to communicate about it. Chest 2018;153:1004-15.

33. Slatore CG, Horeweg N, Jett JR, Midthun DE, Powell CA, Wiener RS, et al. An official American Thoracic Society research statement: a research framework for pulmonary nodule evaluation and management. Am J Respir Crit Care Med 2015;192:500-14.

34. Zeiger MA, Thompson GB, Duh QY, Hamrahian AH, Angelos $P$, Elaraj D, et al. The American Association of Clinical Endocrinologists and American Association of Endocrine Surgeons medical guidelines for the management of adrenal incidentalomas. Endocr Pract 2009;15(Suppl 1):1-20.

35. Massad LS, Einstein MH, Huh WK, Katki HA, Kinney WK, Schiffman M, et al. 2012 updated consensus guidelines for the management of abnormal cervical cancer screening tests and cancer precursors. Obstet Gynecol 2013;121:829-46. 